\title{
The modulating effect of light intensity on the response of the coccolithophore Gephyrocapsa oceanica to ocean acidification
}

\author{
Yong Zhang, ${ }^{* 1}$ Lennart T. Bach, $^{1}$ Kai G. Schulz, ${ }^{2}$ Ulf Riebesell ${ }^{1}$ \\ ${ }^{1}$ Biological Oceanography, GEOMAR Helmholtz-Centre for Ocean Research Kiel, Kiel, Germany \\ ${ }^{2}$ Centre for Coastal Biogeochemistry, School of Environmental Science and Management, Southern Cross University, \\ Lismore, New South Wales, Australia
}

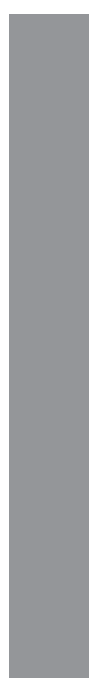

\begin{abstract}
Global change leads to a multitude of simultaneous modifications in the marine realm among which shoaling of the upper mixed layer, leading to enhanced surface layer light intensities, as well as increased carbon dioxide $\left(\mathrm{CO}_{2}\right)$ concentration are some of the most critical environmental alterations for phytoplankton. In this study, we investigated the responses of growth, photosynthetic carbon fixation and calcification of the coccolithophore Gephyrocapsa oceanica to elevated $\mathrm{P}_{\mathrm{CO}_{2}}(51 \mathrm{~Pa}, 105 \mathrm{~Pa}$, and $152 \mathrm{~Pa})(1 \mathrm{~Pa} \approx 10 \mu \mathrm{atm})$ at a variety of light intensities $\left(50-800 \mu \mathrm{mol}\right.$ photons $\left.\mathrm{m}^{-2} \mathrm{~s}^{-1}\right)$. By fitting the light response curve, our results showed that rising $\mathrm{P}_{\mathrm{CO}_{2}}$ reduced the maximum rates for growth, photosynthetic carbon fixation and calcification. Increasing light intensity enhanced the sensitivity of these rate responses to $\mathrm{P}_{\mathrm{CO}_{2}}$, and shifted the $\mathrm{P}_{\mathrm{CO}_{2}}$ optima toward lower levels. Combining the results of this and a previous study (Sett et al. 2014) on the same strain indicates that both limiting low $\mathrm{P}_{\mathrm{CO}_{2}}$ and inhibiting high $\mathrm{P}_{\mathrm{CO}_{2}}$ levels (this study) induce similar responses, reducing growth, carbon fixation and calcification rates of G. oceanica. At limiting low light intensities the $\mathrm{P}_{\mathrm{CO}_{2}}$ optima for maximum growth, carbon fixation and calcification are shifted toward higher levels. Interacting effects of simultaneously occurring environmental changes, such as increasing light intensity and ocean acidification, need to be considered when trying to assess metabolic rates of marine phytoplankton under future ocean scenarios.
\end{abstract}

Atmospheric carbon dioxide $\left(\mathrm{CO}_{2}\right)$ concentrations are projected to increase from about $40 \mathrm{~Pa}(1 \mathrm{~Pa} \approx 10 \mu \mathrm{atm})$ in 2013 beyond $75 \mathrm{~Pa}$ by the end of this century (IPCC 2013). Until today about one third of all anthropogenic $\mathrm{CO}_{2}$ emissions have been absorbed by the ocean (Sabine et al. 2004). Increasing seawater $\mathrm{CO}_{2}$ forms carbonic acid leading to a reduction in seawater $\mathrm{pH}$. The $\mathrm{pH}$ of oceanic surface seawater is projected to decrease by $0.3-0.4$ units within the next 100 yr (Houghton et al. 2001), representing a 100$150 \%$ increase in the proton concentration $\left(\left[\mathrm{H}^{+}\right]\right)$. These changes in $\mathrm{CO}_{2}$ and $\left[\mathrm{H}^{+}\right]$can have positive effects for some phytoplankton functional groups while effects can be negative for others (Riebesell 2004).

Global warming, associated with increasing atmospheric $\mathrm{CO}_{2}$ levels, enhances vertical stratification of the water column and decreases mixing between the surface ocean and deeper layers (Bopp et al. 2001). This expected shoaling of the upper mixed layer increases the average light intensity experienced by phytoplankton suspended in this layer (Sarmiento et al. 2004). Elevated light intensity may accelerate

*Correspondence: yzhang@geomar.de growth rates of some phytoplankton groups, while it might be stressful to others (Merico et al. 2004). When solar irradiance exceeds the capacity of common protective mechanisms, growth and electron transport rates of phytoplankton can be reduced (Gao et al. 2012). Depending on their photosynthetic apparatus, phytoplankton differ in their ability to cope with excess light intensities (Kaeriyama et al. 2011).

Most microalgae have developed energetically costly $\mathrm{CO}_{2}-$ concentrating mechanisms (CCMs) to avoid inorganic carbon limitation at the site of fixation (Giordano et al. 2005). CCMs involve the active uptake of $\mathrm{CO}_{2}$ and/or $\mathrm{HCO}_{3}^{-}$into the algal cell and/or the chloroplast. Given that the operation of CCMs is energetically costly, light availability may affect the activity of CCMs and the activity of CCMs may affect the energy reallocation in phytoplankton (Giordano et al. 2005). Energy saved from the down-regulation of CCMs in response to elevated $\mathrm{CO}_{2}$ permits utilization in other processes such as growth or enzyme synthesis (Schippers et al. 2004; McCarthy et al. 2012).

Coccolithophores play an important role in the marine carbon cycle through the fixation of inorganic carbon via photosynthesis, as well as the precipitation of calcium 
carbonate (Rost and Riebesell 2004). Coccolith formation has been suggested to reduce the risk of photo-damage of coccolithophores under high light conditions either by shading the cells like a sunshade (Braarud et al. 1952) or by contributing to excess energy dissipation (Barcelos e Ramos et al. 2012; Xu and Gao 2012). Declining pH generally reduces calcification rates (Bach et al. 2011; Riebesell and Tortell 2011), which may then put the cells at higher risk to suffer from photo-inhibition.

In this study, we investigated the combined effects of three $\mathrm{P}_{\mathrm{CO}_{2}}$ levels and six light intensities on the cosmopolitan coccolithophore Gephyrocapsa oceanica. We measured the relative electron transport rate (rETR), growth rate, as well as carbon fixation and calcification rates to assess how light intensity modulates the effect of increasing $\mathrm{P}_{\mathrm{CO}_{2}}$ on these parameters in G. oceanica.

\section{Methods}

\section{Experimental setup}

Gephyrocapsa oceanica (strain RCC 1303, isolated from Arcachon Bay, France in 1999) was grown in artificial seawater (ASW) under dilute batch culture conditions at $20^{\circ} \mathrm{C}$. Light intensities were set to $50 \mu \mathrm{mol}$ photons $\mathrm{m}^{-2} \mathrm{~s}^{-1}, 100$ $\mu$ mol photons $\mathrm{m}^{-2} \mathrm{~s}^{-1}, 200 \mu \mathrm{mol}$ photons $\mathrm{m}^{-2} \mathrm{~s}^{-1}, 400$ $\mu$ mol photons $\mathrm{m}^{-2} \mathrm{~s}^{-1}, 600 \mu \mathrm{mol}$ photons $\mathrm{m}^{-2} \mathrm{~s}^{-1}$, and 800 $\mu$ mol photons $\mathrm{m}^{-2} \mathrm{~s}^{-1}$ of photosynthetically active radiation (PAR) in a RUMED Light Thermostat (Rubarth Apparate $\mathrm{GmbH})$ at a $16: 8 \mathrm{~h}$ light : dark cycle. Light intensities were measured at every position in the light chamber where the bottles were put, using a Li-250A data logger (Li-Cor, Heinz Walz GmbH, Effeltrich).

ASW with a salinity of 35 was prepared according to Kester et al. (1967), but with the addition of $2350 \mu \mathrm{mol} \mathrm{kg} \mathrm{kg}^{-1}$ bicarbonate (as opposed to $2330 \mu \mathrm{mol} \mathrm{kg}{ }^{-1}$ in the original recipe). ASW was enriched with $64 \mu \mathrm{mol} \mathrm{kg}{ }^{-1}$ nitrate $\left(\mathrm{NO}_{3}^{-}\right)$, $4 \mu \mathrm{mol} \mathrm{kg}{ }^{-1}$ phosphate $\left(\mathrm{PO}_{4}^{3-}\right), \mathrm{f} / 8$ concentrations for trace metals and vitamins (Guillard and Ryther 1962), $10 \mathrm{nmol}$ $\mathrm{kg}^{-1} \mathrm{SeO}_{2}$ (Danbara and Shiraiwa 1999), and $2 \mathrm{~mL} \mathrm{~kg}^{-1}$ of sterile filtered $\left(0.2 \mu \mathrm{m}\right.$ pore size, Sartobran ${ }^{\circledR}$ P 300, Sartorius) North Sea water to prevent possible trace metal limitation during culturing. Enriched ASW was aerated for $48 \mathrm{~h}$ at $20^{\circ} \mathrm{C}$ $\left(0.2 \mu \mathrm{m}\right.$ pore size, Midisart ${ }^{\circledR} 2000$ PTFE, Sartorius) with air containing 40, 84 or $112 \mathrm{~Pa} \mathrm{P}_{\mathrm{CO}_{2}}$ (ALPHAGAZ ${ }^{\mathrm{TM}}$ ). The dry air/ $\mathrm{CO}_{2}$ mixture was humidified with Milli-Q water before aeration into the ASW to minimize evaporation. After aeration, the ASW medium was sterile-filtered $(0.2 \mu \mathrm{m}$ pore size, Sartobran ${ }^{\circledR}$ P 300, Sartorius) with gentle pressure and carefully pumped into autoclaved $0.5 \mathrm{~L}$ or $2 \mathrm{~L}$ polycarbonate bottles (Nalgene ${ }^{\circledR}$ Bottles). Samples to assess carbonate chemistry conditions at the beginning of the experiment (total alkalinity (TA) and dissolved inorganic carbon (DIC) analysis) were taken from the sterile-filtered medium. $0.5 \mathrm{~L}$ bottles were used to acclimate cells to experimental conditions for
7-9 generations (one replicate, maximum final cell number in these acclimation cultures were 23,000 cells $\mathrm{mL}^{-1}$ ). Depending on growth rate, acclimation time was between 9 (slowest growth) and 4 (fastest growth) days. The main experiment culture was conducted in $2 \mathrm{~L}$ bottles (four replicates). The initial cell concentrations in the main experiment culture and in the pre-culture were about 220 cells $\mathrm{mL}^{-1}$. Bottles for both the acclimation culture and the main experiment were filled with ASW medium leaving a minimum headspace of less than $1 \%$ to keep gas exchange at a minimum. Cells were transferred from $0.5 \mathrm{~L}$ to $2 \mathrm{~L}$ bottles at the same time. The volume of the inoculum was calculated (see below) and the same volume of ASW was taken out from $2 \mathrm{~L}$ bottles before inoculation. All culture bottles were stored at the experimental temperature of $20^{\circ} \mathrm{C}$ for 3 or $4 \mathrm{~d}$ prior to inoculation. Culture bottles were manually rotated twice a day at $5 \mathrm{~h}$ and $12 \mathrm{~h}$ after the onset of the light phase to reduce sedimentation of the cells.

\section{Carbonate chemistry measurements}

Samplings started $3 \mathrm{~h}$ after the onset of the light period and lasted no longer than $2 \mathrm{~h}$. Dissolved inorganic carbon (DIC) samples were sterile filtered $(0.2 \mu \mathrm{m}$ pore size, Filtropur S 0.2, Sarstedt) by gentle pressure into $50 \mathrm{~mL}$ Duran Winkler flasks (Schott). The bottles were filled with samples from bottom to top and with overflow, tightly closed without headspace, and stored at $4^{\circ} \mathrm{C}$. DIC concentrations were measured by an infrared $\mathrm{CO}_{2}$ analyzer system (Automated Infra Red Inorganic Carbon Analyzer, Marianda). Samples for total alkalinity (TA) measurements were filtered with GF/F filters (0.7 $\mu \mathrm{m}$ nominal pore size, Whatman), poisoned with a saturated $\mathrm{HgCl}_{2}$ solution $(0.5 \%$ final concentration), and stored at $4^{\circ} \mathrm{C}$. TA was measured in duplicate by open-cell potentiometric titration using a 862 Compact Titrosample (Metrohm) according to Dickson et al. (2003). DIC and TA samples were collected and measured before and at the end of incubations. Measurements of DIC and TA were corrected with certified reference material (Batch 115, Prof. A. Dickson, La Jolla, California). The carbonate system was calculated from TA, DIC, phosphate, temperature, and salinity using the CO2 System Calculations in MS Excel software (Pierrot et al. 2006) with temperature and salinity dependent stoichiometric equilibrium constants $K_{1}$ and $K_{2}$ for carbonic acid taken from Roy et al. (1993).

\section{Photosynthetic measurements}

The effective quantum yield of photosystem II (PSII) of algae samples was assessed using a Phytoplankton Analyzer PHYTO-PAM (Heinz Walz GmbH) $5 \mathrm{~h}$ after the onset of the light phase. Samples were kept in the dark for $15 \mathrm{~min}$ at room temperature (about $20^{\circ} \mathrm{C}$ ). Gain setting was adjusted with algae sample via the Auto-Gain function and the effect of background signal was suppressed with the help of the Zero Offset function with filtered culture water. PAR levels between $1 \mu \mathrm{mol}$ photons $\mathrm{m}^{-2} \mathrm{~s}^{-1}$ and $1659 \mu \mathrm{mol}$ photons 
$\mathrm{m}^{-2} \mathrm{~s}^{-1}$ were applied in 14 steps of $30 \mathrm{~s}$ each in light response curve measurements.

The relative electron transport rate (rETR) was calculated according to Schreiber et al. (1995), where:

$\mathrm{rETR}=$ Yield $\times$ PAR $\times 0.5 \times 0.84\left(\mu \mathrm{mol}\right.$ electrons $\left.\mathrm{m}^{-2} \mathrm{~s}^{-1}\right)$

where yield $\left(F_{\mathrm{v}} / F_{\mathrm{m}}\right)$ is defined as the ratio of photons emitted to photons absorbed by PSII (Schreiber et al. 1995). Implicit in this equation is the assumption that half of the quanta of the incident PAR are distributed to PSII and 84\% of incident PAR is absorbed by photosynthetic pigments in a standard leaf (Björkman and Demmig 1987).

Photosynthesis vs. irradiance curves (P-I curves) were obtained by plotting calculated rETR vs. corresponding PAR values. P-I curve fitting was performed using a theoretical light response function according to a modified version of the photosynthesis model of Eilers and Peeters (1988).

$$
y=\frac{\mathrm{PAR}}{a \times \mathrm{PAR}^{2}+b \times \mathrm{PAR}+c}
$$

where the coefficients $a, b$ and $c$ are fitted in a least square manner. The model of Eilers and Peeters can be easily interpreted algebraically. At low light intensity, $b \times$ PAR and $a \times$ $\mathrm{PAR}^{2}$ can be neglected and $y$ (ETR) increases approximately linearly with light intensity. At high light intensity, $a \times$ $\mathrm{PAR}^{2}$ dominates and thus $y$ (ETR) is inversely proportional to the light intensity. The initial slope of the light limited part of the P-I curve constitutes a measure of the quantum yield of electron transport, indicated as alpha, which was calculated as:

$$
\text { alpha }=\frac{1}{c}
$$

The maximum value $\left(Y_{\max }\right)$ of rETR was calculated as:

$$
Y_{\max }=\frac{1}{b+2 \sqrt{a c}}
$$

Here, $Y_{\max }$ shows the saturation level of $\mathrm{rETR}\left(\mathrm{rETR}_{\max }\right)$. Saturation light intensity $I_{\mathrm{k}}$ corresponds to the PAR value at the crossing point of the lines defined by the initial slope and $\mathrm{rETR}_{\max } . I_{\mathrm{k}}$ is calculated from the expression $\mathrm{rETR}_{\max } / \mathrm{alpha}$ and is characteristic for the onset of light saturation.

\section{Growth rate measurements}

At the end of incubations, about $25 \mathrm{~mL}$ samples were taken from the culture bottles at the same time, $\sim 7 \mathrm{~h}$ after the onset of the light phase. Cell numbers were determined using a Z2 Coulter Particle Counter and Size Analyzer (Beckman). Growth rate $(\mu)$ was calculated for each replicate according to the equation:

$$
\mu=\left(\ln N_{1}-\ln N_{0}\right) / d
$$

where $N_{0}$ and $N_{1}$ are cell numbers at the beginning and the end of a growth interval, and $d$ is the duration of the growth period in days.

\section{Particulate organic (POC) and inorganic carbon (PIC) measurements}

Samples for total particulate carbon (TPC) and particulate organic carbon (POC) were gently filtered (200 mbar) onto pre-combusted $\left(500^{\circ} \mathrm{C}, 8 \mathrm{~h}\right) \mathrm{GF} / \mathrm{F}$ filters and stored in the dark at $-20^{\circ} \mathrm{C}$. Prior to the measurement, POC filters were fumed with $37.1 \% \mathrm{HCl}(\mathrm{w} / \mathrm{w})$ for $2 \mathrm{~h}$ to remove all inorganic carbon. After $8 \mathrm{~h}$ of drying at $60^{\circ} \mathrm{C}$, TPC and POC were measured using an isotope ratio mass spectrometer (Thermo Finnigan MAT $253 \mathrm{GmbH}$ ). Particulate inorganic carbon (PIC) was calculated as the difference between TPC and POC. POC and PIC production rates were calculated as:

$$
\text { POC production rate }=\mu\left(\mathrm{d}^{-1}\right) \times \text { POC content }\left(\text { pg C cell }{ }^{-1}\right)
$$

PIC production rate $=\mu\left(\mathrm{d}^{-1}\right) \times$ PIC content $\left(\right.$ pg C cell $\left.{ }^{-1}\right)$

\section{Data analysis}

Growth, POC and PIC production rates as a function of light intensity (PAR) were fitted at each $\mathrm{P}_{\mathrm{CO}_{2}}$ level $(51 \mathrm{~Pa}$, $105 \mathrm{~Pa}$, and $152 \mathrm{~Pa}$ ) with the model of Eilers and Peeters (1988) (Eq. 2). The theoretical maximum rates for growth, POC and PIC production are calculated according to Eq. 4. Conversely, we fitted growth, POC and PIC production rates at each light intensity as a function of $\mathrm{P}_{\mathrm{CO}_{2}}$ using the modified Michaelis-Menten equation:

$$
y=\frac{X \times \mathrm{P}_{\mathrm{CO}_{2}}}{Y+\mathrm{P}_{\mathrm{CO}_{2}}}-s \times \mathrm{P}_{\mathrm{CO}_{2}}
$$

derived by Bach et al. (2011). Here, $s$ is the constant which describes the negative effect of increasing $\left[\mathrm{H}^{+}\right]$(which is quasi proportional to $\mathrm{P}_{\mathrm{CO}_{2}}$ at constant TA). $y$ is growth, POC or PIC production rate at a certain $\mathrm{P}_{\mathrm{CO}_{2}}$ level. $X$ and $Y$ are random fit parameters which can be converted to the Michaelis-Menten parameters $V_{\max }$ and $K_{1 / 2}$ with a mathematical procedure described in Bach et al. (2011). The underlying assumption implicit in this equation is that growth, POC and PIC production rates follow an optimum curve over a broad range of $\mathrm{P}_{\mathrm{CO}_{2}}$ values at constant TA, which has been shown for a variety of coccolithophore species (Langer et al. 2006; Bach et al. 2011; Sett et al. 2014; Bach et al. 2015).

The effect of the $\mathrm{P}_{\mathrm{CO}_{2}}$ treatment on $V_{\max }$ was determined by means of a one-way analysis of variance (ANOVA). A twoway ANOVA was used to determine the main effect of $\mathrm{P}_{\mathrm{CO}_{2}}$ and light treatments and their interactions for these 
Table 1. Carbonate system parameters of the artificial seawater. DIC and TA samples were collected and measured before and at the end of incubations. The carbonate system parameters were calculated from TA, DIC, phosphate concentration $\left(4 \mu \mathrm{mol}^{\mathrm{kg}}{ }^{-1}\right)$, temperature $\left(20^{\circ} \mathrm{C}\right)$, and salinity (35) using the $\mathrm{CO}_{2}$ System Calculations in MS Excel software (Pierrot et al. 2006).

\begin{tabular}{|c|c|c|c|c|c|c|c|}
\hline $\mathrm{P}_{\mathrm{CO}_{2}} \mathrm{~Pa}$ & TA $\mu \mathrm{mol} \mathrm{kg}^{-1}$ & DIC $\mu \mathrm{mol} \mathrm{kg}{ }^{-1}$ & $\mathrm{pH}$ total scale & $\mathrm{HCO}_{3}^{-} \mu \mathrm{mol} \mathrm{kg}{ }^{-1}$ & $\mathrm{CO}_{3}^{2-} \mu \mathrm{mol} \mathrm{kg}{ }^{-1}$ & $\mathrm{CO}_{2} \mu \mathrm{mol} \mathrm{kg}{ }^{-1}$ & $\Omega$ calcite \\
\hline $105 \pm 9^{b}$ & $2325 \pm 14^{b}$ & $2213 \pm 19^{a}$ & $7.69 \pm 0.04^{b}$ & $2080 \pm 22^{b}$ & $100 \pm 8^{b}$ & $34.1 \pm 3.3^{b}$ & $2.4 \pm 0.2^{\mathrm{b}}$ \\
\hline
\end{tabular}

Characters $a, b, c$ represent statistically different means between different $\mathrm{P}_{\mathrm{CO}_{2}}$ treatments (Tukey Post hoc, $p<0.001$ ). The values are expressed as mean values with standard deviation calculated from measurements before and at the end of incubations.

variables. A Tukey Post hoc test was performed to identify the source of the main effect determined by ANOVA. Normality of residuals was tested with a Shapiro-Wilk's test. Levene's test was conducted graphically to test for homogeneity of variances in case of significant data. A generalized least squares (GLS) model was used to stabilize heterogeneity if variances were inhomogeneous. All statistical calculations were performed using $R$ version 2.15.2.

\section{Results}

Carbonate chemistry

All parameters (measured and calculated) of the carbonate system are presented in Table 1 . Air pressure in the headspace of the $10 \mathrm{~L}$ bubbling bottles was about 25-35\% higher than one standard atmosphere, leading to higher $\mathrm{P}_{\mathrm{CO}_{2}}$ levels in air-saturated ASW than targeted $\mathrm{P}_{\mathrm{CO}_{2}}$. After aerating for $48 \mathrm{~h}$ at about 40,84 , and $112 \mathrm{~Pa} \mathrm{P}_{\mathrm{CO}_{2}}$, the $\mathrm{P}_{\mathrm{CO}_{2}}$ levels of the

Table 2. Results of two-way ANOVAs of the effects of $\mathrm{PCO}_{\mathrm{CO}_{2}}$, light intensity (PAR) and their interaction on $\mu$, rETR $\mathrm{max}_{\mathrm{m}}$ alpha, $I_{\mathrm{k}}$, POC and PIC production rates, PIC : POC ratio, POC : PON ratio.

\begin{tabular}{|c|c|c|c|c|}
\hline Parameter & Treatment & df & F value & $p$ value \\
\hline \multirow[t]{3}{*}{$\mu$} & $\mathrm{P}_{\mathrm{CO}_{2}}$ & 2 & 3928.30 & $<0.001$ \\
\hline & PAR & 5 & 18551.90 & $<0.001$ \\
\hline & $\mathrm{P}_{\mathrm{CO}_{2}} \times \mathrm{PAR}$ & 10 & 1651.00 & $<0.001$ \\
\hline \multirow[t]{3}{*}{$\mathrm{rETR}_{\max }$} & $\mathrm{P}_{\mathrm{CO}_{2}}$ & 2 & 1544.10 & $=0.003$ \\
\hline & PAR & 5 & 1025.51 & $<0.001$ \\
\hline & $\mathrm{P}_{\mathrm{CO}_{2}} \times \mathrm{PAR}$ & 10 & 25.89 & $<0.001$ \\
\hline \multirow[t]{3}{*}{ alpha } & $\mathrm{P}_{\mathrm{CO}_{2}}$ & 2 & 21.80 & $<0.001$ \\
\hline & PAR & 5 & 644.00 & $<0.001$ \\
\hline & $\mathrm{P}_{\mathrm{CO}_{2}} \times \mathrm{PAR}$ & 10 & 46.80 & $<0.001$ \\
\hline \multirow[t]{3}{*}{$l_{\mathrm{k}}$} & $\mathrm{P}_{\mathrm{CO}_{2}}$ & 2 & 883.28 & $<0.001$ \\
\hline & PAR & 5 & 3312.69 & $<0.001$ \\
\hline & $\mathrm{P}_{\mathrm{CO}_{2}} \times \mathrm{PAR}$ & 10 & 122.17 & $<0.001$ \\
\hline \multirow[t]{3}{*}{$\mathrm{POC}$ production rate } & $\mathrm{P}_{\mathrm{CO}_{2}}$ & 2 & 9174.71 & $<0.001$ \\
\hline & PAR & 5 & 3738.19 & $<0.001$ \\
\hline & $\mathrm{P}_{\mathrm{CO}_{2}} \times \mathrm{PAR}$ & 10 & 55.36 & $<0.001$ \\
\hline \multirow[t]{3}{*}{ PIC production rate } & $\mathrm{P}_{\mathrm{CO}_{2}}$ & 2 & 346.08 & $<0.001$ \\
\hline & PAR & 5 & 857.79 & $<0.001$ \\
\hline & $\mathrm{P}_{\mathrm{CO}_{2}} \times \mathrm{PAR}$ & 10 & 107.97 & $<0.001$ \\
\hline \multirow[t]{3}{*}{ PIC : POC ratio } & $\mathrm{P}_{\mathrm{CO}_{2}}$ & 2 & 627.001 & $<0.001$ \\
\hline & PAR & 5 & 28.994 & $<0.001$ \\
\hline & $\mathrm{P}_{\mathrm{CO}_{2}} \times \mathrm{PAR}$ & 10 & 16.675 & $<0.001$ \\
\hline \multirow[t]{3}{*}{ POC : PON ratio } & $\mathrm{P}_{\mathrm{CO}_{2}}$ & 2 & 20.46 & $<0.001$ \\
\hline & PAR & 5 & 19.85 & $<0.001$ \\
\hline & $\mathrm{P}_{\mathrm{CO}_{2}} \times \mathrm{PAR}$ & 10 & 2.71 & $=0.009$ \\
\hline
\end{tabular}

PAR, photosynthetically active radiation ( $\mu$ mol photons $\left.\mathrm{m}^{-2} \mathrm{~s}^{-1}\right) ; \mu$, growth rate $\left(\mathrm{d}^{-1}\right) ; \mathrm{rETR}_{\max }$, maximum relative electron transport rate $(\mu \mathrm{mol}$ electrons $\mathrm{m}^{-2} \mathrm{~s}^{-1}$ ); alpha, slope of the light-limited part of the photosynthesis versus irradiance curve; $l_{\mathrm{k}}$, saturating photon flux density ( $\mu$ mol photons $\left.\mathrm{m}^{-2} \mathrm{~s}^{-1}\right)$; POC production rate, particulate organic carbon production rate (pg C cell ${ }^{-1} \mathrm{~d}^{-1}$ ); PIC production rate, particulate inorganic carbon production rate $\left(\mathrm{pgC} \mathrm{Cell}^{-1} \mathrm{~d}^{-1}\right)$. 
ASW were about $51 \mathrm{~Pa}, 105 \mathrm{~Pa}$, and $152 \mathrm{~Pa}$, and resulting $\mathrm{pH}_{\mathrm{T}}$ (reported on the total scale) were about 7.96, 7.69, and 7.54, respectively.

\section{Growth rates}

Light intensities and $\mathrm{P}_{\mathrm{CO}_{2}}$ levels significantly affected growth rates in $G$. oceanica, both individually as well as interactively (Table 2). At a $\mathrm{P}_{\mathrm{CO}_{2}}$ of $51 \mathrm{~Pa}$, growth rates of G. oceanica increased with increasing light intensity until 800 $\mu$ mol photons $\mathrm{m}^{-2} \mathrm{~s}^{-1}$. At higher $\mathrm{P}_{\mathrm{CO}_{2}}$ levels, however, growth rates only increased until $400 \mu \mathrm{mol}$ photons $\mathrm{m}^{-2} \mathrm{~s}^{-1}$ and slightly declined thereafter (Fig. 1A).

At $50 \mu \mathrm{mol}$ photons $\mathrm{m}^{-2} \mathrm{~s}^{-1}$, growth rates of G. oceanica were similar at the three $\mathrm{P}_{\mathrm{CO}_{2}}$ levels (Tukey Post hoc, $p>0.1$ ). At $100 \mu \mathrm{mol}$ photons $\mathrm{m}^{-2} \mathrm{~s}^{-1}$, growth rate at $105 \mathrm{~Pa} \mathrm{P}_{\mathrm{CO}_{2}}$ was higher than at 51 and $152 \mathrm{~Pa} \mathrm{P}_{\mathrm{CO}_{2}}$ (Tukey Post hoc, $p<0.001$ ) (Fig. 1; Table 3). At $200 \mu \mathrm{mol}$ photons $\mathrm{m}^{-2} \mathrm{~s}^{-1}$ and above, growth rates decreased with elevated $\mathrm{P}_{\mathrm{CO}_{2}}$ levels (Tukey Post hoc, all df $=2$, all $p<0.001$ ) (Fig. 1; Table 3). Fitted maximum growth rates declined significantly with rising $\mathrm{P}_{\mathrm{CO}_{2}}$ levels (one-way ANOVA, $F=3836$, df $=2, p<0.001$; Tukey Post hoc, $\mathrm{df}=2, p<0.001$ ) (Fig. 1A; Table 4).

\section{rETR $_{\text {max }}$, alpha and $I_{k}$}

We identified statistically significant effects of light intensity, $\mathrm{P}_{\mathrm{CO}_{2}}$ level and their interaction also on $\mathrm{rETR}_{\max }$, alpha and $I_{\mathrm{k}}$ (Table 2). $\mathrm{rETR}_{\max }$ followed the same pattern as described for growth rate in the previous section. At $51 \mathrm{~Pa}$, $\mathrm{rETR}_{\max }$ of $G$. oceanica increased with increasing light radiation until $800 \mu \mathrm{mol}$ photons $\mathrm{m}^{-2} \mathrm{~s}^{-1}$. At higher $\mathrm{P}_{\mathrm{CO}_{2}}$ levels, however, $\mathrm{rETR}_{\max }$ increased only until $400 \mu \mathrm{mol}$ photons $\mathrm{m}^{-2} \mathrm{~s}^{-1}$ or $600 \mu \mathrm{mol}$ photons $\mathrm{m}^{-2} \mathrm{~s}^{-1}$ and decreased significantly thereafter (Fig. 2A; Table 3). At $50 \mu \mathrm{mol}$ photons $\mathrm{m}^{-2}$ $\mathrm{s}^{-1}, 100 \mu \mathrm{mol}$ photons $\mathrm{m}^{-2} \mathrm{~s}^{-1}$, and $200 \mu \mathrm{mol}$ photons $\mathrm{m}^{-2}$ $\mathrm{s}^{-1}, \mathrm{rETR}_{\max }$ did not show any significant differences among the three $\mathrm{P}_{\mathrm{CO}_{2}}$ treatments (Tukey Post hoc, all $\mathrm{df}=2$, all $p>0.05)$.

Increasing light intensity resulted in a relatively constant decrease in alpha (Fig. 2B). The decline of alpha from lowest to highest light intensities was 23\%, 32\%, and 57\% for $51 \mathrm{~Pa}, 105 \mathrm{~Pa}$, and $152 \mathrm{~Pa}$, respectively (Tukey Post hoc, all $\mathrm{df}=1$, all $p<0.001$ ) (Fig. $2 \mathrm{~B}$ ). At $50 \mu \mathrm{mol}$ photons $\mathrm{m}^{-2} \mathrm{~s}^{-1}$ or $100 \mu \mathrm{mol}$ photons $\mathrm{m}^{-2} \mathrm{~s}^{-1}$, alpha was not significantly different among the three $\mathrm{P}_{\mathrm{CO}_{2}}$ treatments (Tukey Post hoc, both $\mathrm{df}=2$, both $p>0.05$ ). At $200 \mu \mathrm{mol}$ photons $\mathrm{m}^{-2} \mathrm{~s}^{-1}$ or $400 \mu \mathrm{mol}$ photons $\mathrm{m}^{-2} \mathrm{~s}^{-1}$, alpha at $105 \mathrm{~Pa} \mathrm{P}_{\mathrm{CO}_{2}}$ was lower than at 51 and $152 \mathrm{~Pa} \mathrm{P}_{\mathrm{CO}_{2}}$ (Tukey Post hoc, both $p<0.01)$. At $800 \mu$ mol photons $\mathrm{m}^{-2} \mathrm{~s}^{-1}$, alpha decreased significantly with elevated $\mathrm{P}_{\mathrm{CO}_{2}}$ treatments (Tukey Post hoc, both $p<0.01$ ). It seems that effects of $\mathrm{P}_{\mathrm{CO}_{2}}$ levels on alpha were amplified by increasing light intensity (Fig. 2B).

Saturation light intensity, $I_{\mathrm{k}}$, more than doubled from lowest to highest light intensities. With light intensity increasing from $50 \mu \mathrm{mol}$ photons $\mathrm{m}^{-2} \mathrm{~s}^{-1}$ to $800 \mu \mathrm{mol}$ pho-
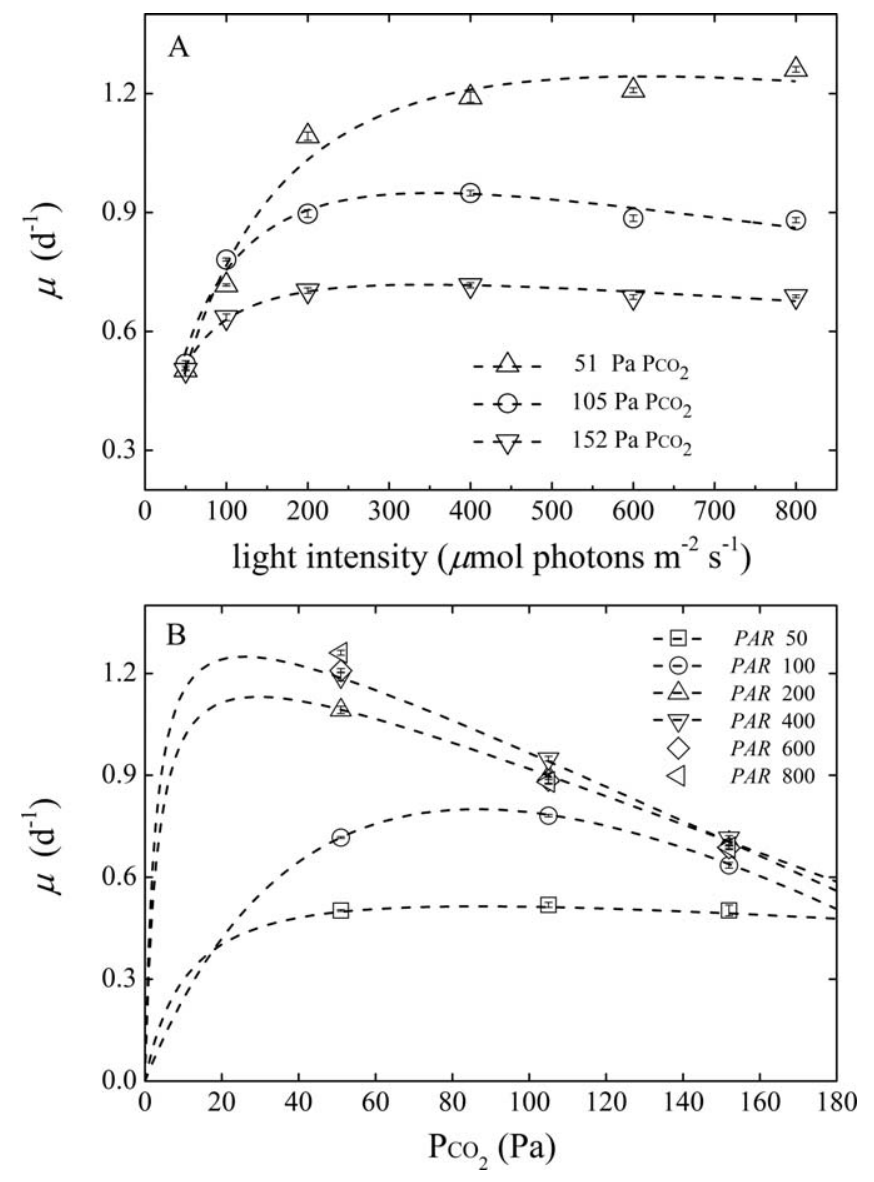

Fig. 1. Effects of light intensity and $\mathrm{P}_{\mathrm{CO}_{2}}$ level on growth rate of Gephyrocapsa oceanica. (A) Growth rate as a function of light intensities at $51(\triangle), 105(\bigcirc)$ and $152(\nabla) \mathrm{Pa} \mathrm{P}_{\mathrm{CO}_{2}}$. (B) Growth rate as a function of $\mathrm{P}_{\mathrm{CO}_{2}}$ levels at light intensities of $50 \mu \mathrm{mol}$ photons $\mathrm{m}^{-2} \mathrm{~s}^{-1}, 100 \mu \mathrm{mol}$ photons $\mathrm{m}^{-2} \mathrm{~s}^{-1}, 200 \mu \mathrm{mol}$ photons $\mathrm{m}^{-2} \mathrm{~s}^{-1}, 400 \mu \mathrm{mol}$ photons $\mathrm{m}^{-2}$ $\mathrm{s}^{-1}, 600 \mu \mathrm{mol}$ photons $\mathrm{m}^{-2} \mathrm{~s}^{-1}$ and $800 \mu$ mol photons $\mathrm{m}^{-2} \mathrm{~s}^{-1}$. Dashed lines in panel (A) were fitted using Eq. 2. Dashed lines in panel (B) were fitted using Eq. 8. The values represent the mean of four replicates, with error bars showing \pm one standard deviation. Please note that based on only three points, growth rate response curves at 600 $\mu \mathrm{mol}$ photons $\mathrm{m}^{-2} \mathrm{~s}^{-1}$ and $800 \mu \mathrm{mol}$ photons $\mathrm{m}^{-2} \mathrm{~s}^{-1}$ in panel (B) cannot be fitted using Eq. 8 .

tons $\mathrm{m}^{-2} \mathrm{~s}^{-1}, I_{\mathrm{k}}$ increased about 2.2, 2.4, and 3.5 times for $51 \mathrm{~Pa}, 105 \mathrm{~Pa}$, and $152 \mathrm{~Pa}$, respectively (Tukey Post hoc, $\mathrm{df}=1, p<0.001$ ) (Fig. 2C; Table 3). At $50 \mu \mathrm{mol}$ photons $\mathrm{m}^{-2}$ $\mathrm{s}^{-1}, 100 \mu \mathrm{mol}$ photons $\mathrm{m}^{-2} \mathrm{~s}^{-1}$, or $200 \mu \mathrm{mol}$ photons $\mathrm{m}^{-2}$ $\mathrm{s}^{-1}, I_{\mathrm{k}}$ did not show significant difference among the three $\mathrm{P}_{\mathrm{CO}_{2}}$ treatments (Tukey Post hoc, all $\mathrm{df}=2$, all $p>0.1$ ). At $400 \mu \mathrm{mol}$ photons $\mathrm{m}^{-2} \mathrm{~s}^{-1}$ or $600 \mu \mathrm{mol}$ photons $\mathrm{m}^{-2} \mathrm{~s}^{-1}, I_{\mathrm{k}}$ at $51 \mathrm{~Pa} \mathrm{P}_{\mathrm{CO}_{2}}$ was lower than at 105 and $152 \mathrm{~Pa} \mathrm{P}_{\mathrm{CO}_{2}}$ treatments (Tukey Post hoc, both $\mathrm{df}=1$, both $p<0.05$ ). At 800 $\mu$ mol photons $\mathrm{m}^{-2} \mathrm{~s}^{-1}, I_{\mathrm{k}}$ increased significantly with elevated $\mathrm{P}_{\mathrm{CO}_{2}}$ (Tukey Post hoc, $\mathrm{df}=2, p<0.01$ ). It seems that the positive effect of $\mathrm{P}_{\mathrm{CO}_{2}}$ treatment on $I_{\mathrm{k}}$ was also amplified by increasing light intensity (Fig. 2C). 
Table 3. Experimental condition, growth rate, photosynthesis parameter and carbon production in dilute batch culture incubation.

\begin{tabular}{|c|c|c|c|c|c|c|c|c|c|}
\hline$\underline{\mathbf{P}_{\mathrm{CO}_{2}}}$ & PAR & $\mu$ & $\mathrm{rETR}_{\max }$ & alpha & $I_{\mathrm{k}}$ & $\mathrm{POC} /$ cell/d & $\mathrm{PIC} /$ cell/d & PIC/POC & POC/PON \\
\hline & 100 & $0.72(0.003)$ & 111 (3) & $0.29(0.001)$ & $381(11)$ & $17.04(0.670)$ & $23.80(2.492)$ & $1.40(0.146)$ & $8.04(0.316)$ \\
\hline & 400 & $1.19(0.013)$ & $143(2)$ & $0.26(0.004)$ & $539(4)$ & $33.85(2.514)$ & $46.58(12.711)$ & $1.38(0.097)$ & $7.22(0.536)$ \\
\hline & 600 & $1.21(0.006)$ & $154(3)$ & $0.23(0.006)$ & $678(14)$ & $32.59(1.028)$ & $52.69(4.065)$ & $1.62(0.125)$ & $7.32(0.231)$ \\
\hline & 800 & $1.26(0.007)$ & 157 (2) & $0.23(0.006)$ & $692(10)$ & $29.47(0.233)$ & $48.78(1.374)$ & $1.66(0.047)$ & $6.90(0.055)$ \\
\hline & 100 & $0.78(0.004)$ & $101(2)$ & $0.29(0.002)$ & $352(6)$ & $17.84(0.722)$ & $17.27(1.472)$ & $0.97(0.825)$ & $6.73(0.273)$ \\
\hline & 200 & $0.90(0.010)$ & $126(2)$ & $0.26(0.004)$ & $487(8)$ & $25.82(3.484)$ & 19.19 (3.577) & $0.74(0.139)$ & $6.91(0.933)$ \\
\hline & 400 & $0.95(0.007)$ & 157 (3) & $0.24(0.003)$ & $643(9)$ & $28.66(4.087)$ & $18.71(4.367)$ & $0.65(0.152)$ & $6.05(0.863)$ \\
\hline & 600 & $0.89(0.008)$ & $180(10)$ & $0.24(0.005)$ & $748(38)$ & $21.16(1.988)$ & $23.35(2.075)$ & $1.10(0.098)$ & $5.99(0.563)$ \\
\hline & 800 & $0.88(0.007)$ & $155(17)$ & $0.20(0.010)$ & $773(71)$ & $19.89(1.391)$ & $21.64(0.777)$ & $1.12(0.040)$ & $6.27(0.451)$ \\
\hline \multirow[t]{3}{*}{152} & 50 & $0.50(0.016)$ & $84(4)$ & $0.28(0.003)$ & $295(11)$ & $8.19(0.077)$ & $5.55(2.157)$ & $0.81(0.013)$ & $6.76(0.052)$ \\
\hline & 600 & $0.69(0.006)$ & $153(7)$ & $0.20(0.006)$ & $773(39)$ & $13.88(1.468)$ & $10.33(1.083)$ & $0.74(0.078)$ & $6.50(0.687)$ \\
\hline & 800 & $0.69(0.004)$ & $130(11)$ & $0.12(0.011)$ & $1028(20)$ & $14.92(2.454)$ & $13.26(0.748)$ & $0.89(0.050)$ & $6.20(1.020)$ \\
\hline
\end{tabular}

$\mathrm{POC} /$ cell/d, particulate organic carbon production rate $\left(\mathrm{pg} \mathrm{C} \mathrm{cell}{ }^{-1} \mathrm{~d}^{-1}\right) ; \mathrm{PIC} /$ cell/d, particulate inorganic carbon production rate $\left(\mathrm{pg} C\right.$ cell $\left.{ }^{-1} \mathrm{~d}^{-1}\right)$; PIC/POC, PIC : POC ratio; POC/PON, POC : PON ratio. More detailed information is given as in Table 2. The values are expressed as the mean of four replicates. Data in the brackets are the standard deviations for four replicates.

\section{POC production rate}

Both, changing carbonate chemistry conditions and light intensity independently and interactively affected POC production rates (Table 2). POC production rates increased significantly at $51 \mathrm{~Pa}$ and $105 \mathrm{~Pa}$ with increasing light intensity until $400 \mu \mathrm{mol}$ photons $\mathrm{m}^{-2} \mathrm{~s}^{-1}$. At $51 \mathrm{~Pa}$, POC production rates did not show a significant difference at 400-800 $\mu \mathrm{mol}$ photons $\mathrm{m}^{-2} \mathrm{~s}^{-1}$ (Tukey Post hoc, $\mathrm{df}=1, p>0.1$ ) (Fig. 3A). At $105 \mathrm{~Pa}$, POC production rates decreased significantly when light intensity increased from $400 \mu \mathrm{mol}$ photons $\mathrm{m}^{-2}$ $\mathrm{s}^{-1}$ to $800 \mu \mathrm{mol}$ photons $\mathrm{m}^{-2} \mathrm{~s}^{-1}$ (Tukey Post hoc, $\mathrm{df}=1$, $p<0.001$ ) (Fig. 3A; Table 3). In comparison to $51 \mathrm{~Pa}$, measured POC production rates at $105 \mathrm{~Pa}_{\mathrm{CO}_{2}}$ were higher at 200

Table 4. Calculated maximum values for growth, $P O C$ and PIC production rates. At each $\mathrm{P}_{\mathrm{CO}_{2}}$ level, growth, $\mathrm{POC}$ and $\mathrm{PIC}$ production rates were fitted using equation 2 , and the maximum values were calculated according to equation 4 .

\begin{tabular}{|c|c|c|c|}
\hline $\mathrm{P}_{\mathrm{CO}_{2}}(\mathrm{~Pa})$ & $\begin{array}{c}\text { Maximum } \\
\text { growth } \\
\text { rate }\left(d^{-1}\right)\end{array}$ & $\begin{array}{l}\text { Maximum } \mathrm{POC} \\
\text { production rate } \\
\left(\mathrm{pgC} \mathrm{cell}^{-1} \mathrm{~d}^{-1}\right)\end{array}$ & $\begin{array}{c}\text { Maximum PIC } \\
\text { production rate } \\
\left(\text { pg C cell }{ }^{-1} d^{-1}\right)\end{array}$ \\
\hline 51 & $1.24 \pm 0.01^{\mathrm{a}}$ & $34.42 \pm 2.65^{\mathrm{a}}$ & $49.90 \pm 2.59^{a}$ \\
\hline 105 & $0.95 \pm 0.01^{b}$ & $29.33 \pm 4.30^{\mathrm{a}}$ & $22.44 \pm 1.44^{b}$ \\
\hline 152 & $0.72 \pm 0.01^{c}$ & $14.72 \pm 0.94^{b}$ & $12.78 \pm 0.64^{c}$ \\
\hline
\end{tabular}

Different letters represent statistical different means (Tukey Post hoc, $p<0.001)$. The values are expressed as the mean of four replicates \pm one standard deviation. $\mu$ mol photons $\mathrm{m}^{-2} \mathrm{~s}^{-1}$ (Tukey Post hoc, $\mathrm{df}=1, p<0.01$ ), but significantly lower at $400 \mu \mathrm{mol}$ photons $\mathrm{m}^{-2} \mathrm{~s}^{-1}, 600 \mu \mathrm{mol}$ photons $\mathrm{m}^{-2} \mathrm{~s}^{-1}$, and $800 \mu \mathrm{mol}$ photons $\mathrm{m}^{-2} \mathrm{~s}^{-1}$ (Fig. $3 \mathrm{~A}, \mathrm{~B})$.

At $152 \mathrm{~Pa}$, POC production rates increased with enhanced light radiation until $200 \mu \mathrm{mol}$ photons $\mathrm{m}^{-2} \mathrm{~s}^{-1}$ (Tukey Post hoc, $\mathrm{df}=1, p<0.05)$ and levelled off with further increases in light intensity (Tukey Post hoc, $\mathrm{df}=3, p>0.05$ ) (Fig. 3A). At $50 \mu \mathrm{mol}$ photons $\mathrm{m}^{-2} \mathrm{~s}^{-1}$, POC production rates did not show any difference at the three $\mathrm{P}_{\mathrm{CO}_{2}}$ treatments (Tukey Post hoc, all $\mathrm{df}=2, p>0.1$ ) (Fig. 3A,B). At 100-800 $\mu$ mol photons $\mathrm{m}^{-2} \mathrm{~s}^{-1}$, POC production rates at high $\mathrm{P}_{\mathrm{CO}_{2}}$ were lower than at intermediate $\mathrm{P}_{\mathrm{CO}_{2}}$ (Tukey Post hoc, all $\mathrm{df}=1, p<0.05$ at $100,200,400$, and 600 treatments; $p>0.05$ at 800 treatment). At $400 \mu \mathrm{mol}$ photons $\mathrm{m}^{-2} \mathrm{~s}^{-1}, 600 \mu \mathrm{mol}$ photons $\mathrm{m}^{-2} \mathrm{~s}^{-1}$, and $800 \mu \mathrm{mol}$ photons $\mathrm{m}^{-2} \mathrm{~s}^{-1}$, POC production rates at intermediate $\mathrm{P}_{\mathrm{CO}_{2}}$ were significantly lower than at low $\mathrm{P}_{\mathrm{CO}_{2}}$ (Tukey Post hoc, all $\mathrm{df}=1, p<0.05$ ). Maximum POC production rates at 51 and $105 \mathrm{~Pa}_{\mathrm{CO}_{2}}$ were not significantly different (Tukey Post hoc, $\mathrm{df}=1$, both $p>0.05$ ), and were higher than that at $152 \mathrm{~Pa}_{\mathrm{CO}_{2}}$ (Tukey Post hoc, df $=1$, both $p<0.001$ ) (Table 4).

\section{PIC production rate}

Both, changing carbonate chemistry conditions and light intensity independently and interactively affected PIC production rates (Table 2). At $50 \mu \mathrm{mol}$ photons $\mathrm{m}^{-2} \mathrm{~s}^{-1}$, PIC production rates decreased by about $35 \%$ and $48 \%$ from low to intermediate and high $\mathrm{P}_{\mathrm{CO}_{2}}$ (Tukey Post hoc, both $\mathrm{df}=1$, 

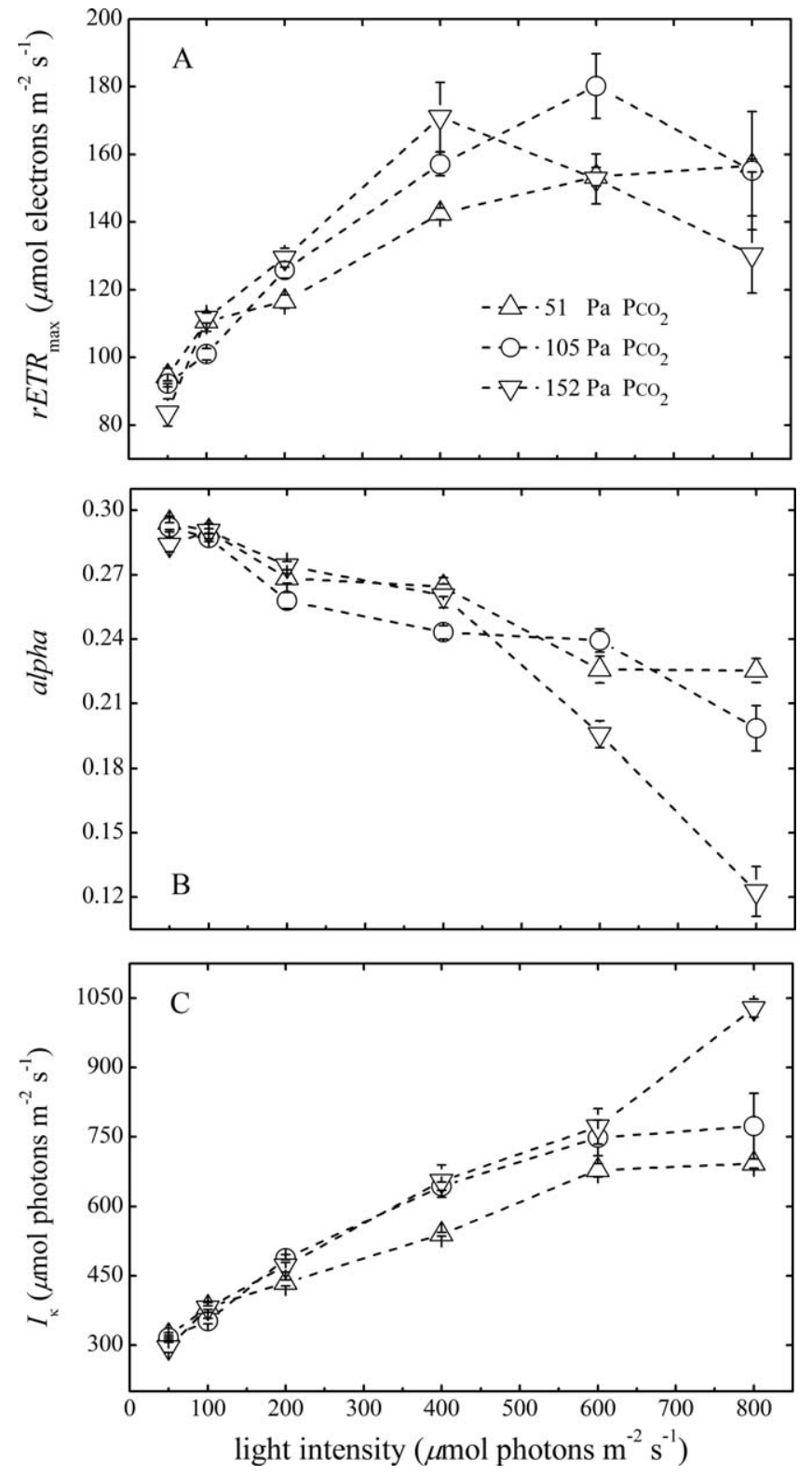

Fig. 2. $\mathrm{rETR}_{\max }$ alpha and $l_{\mathrm{k}}$ of $G$. oceanica as a function of light intensities at $51(\triangle), 105(\bigcirc)$ and $152(\nabla) \mathrm{Pa} \mathrm{P}_{\mathrm{CO}_{2}}$. (A) The maximum of rETR $\left(\mathrm{rETR}_{\max }\right)$ as a function of light intensity. (B) The initial slope of the light limited part of the P-I curve (alpha) as a function of light intensity. (C) Saturation light intensity $\left(I_{k}\right)$ as a function of light intensity. $\mathrm{rETR}_{\max }$ was calculated according to Eq. 4, alpha was calculated with Eq. 3, and $l_{\mathrm{k}}$ was calculated from the expression $\mathrm{rETR}_{\max } /$ alpha. The values represent the mean of four replicates, with error bars representing \pm one standard deviation.

both $p>0.05$ ) (Fig. 3C,D; Table 3). At 100-800 $\mu$ mol photons $\mathrm{m}^{-2} \mathrm{~s}^{-1}$, PIC production rates decreased by $27-56 \%$ at 105 $\mathrm{Pa}$, and by $65-80 \%$ at $152 \mathrm{~Pa}$ in comparison to $51 \mathrm{~Pa}$ (Tukey Post hoc, all $\mathrm{df}=1$, all $p<0.001$ ).

Light intensity had a positive effect on calcification rates at 51 and $105 \mathrm{~Pa} \mathrm{P}_{\mathrm{CO}_{2}}$ levels (Fig. 3C; Table 3). At $51 \mathrm{~Pa}$
$\mathrm{P}_{\mathrm{CO}_{2}}$, calcification rates were enhanced by about 4.5 times with enhanced light radiation until $800 \mu \mathrm{mol}$ photons $\mathrm{m}^{-2}$ $\mathrm{s}^{-1}$. At $105 \mathrm{~Pa} \mathrm{P}_{\mathrm{CO}_{2}}$, PIC production rates only increased until $100 \mu \mathrm{mol}$ photons $\mathrm{m}^{-2} \mathrm{~s}^{-1}$ but stayed constant with a further increase in light intensity (Tukey Post hoc, $\mathrm{df}=4$, all $p>0.05)$. At $152 \mathrm{~Pa}_{\mathrm{CO}_{2}}$, PIC production rates did not show significant differences among six light treatments (Tukey Post hoc, $\mathrm{df}=5$, all $p>0.05$ ). Maximum PIC production rates declined significantly with rising $\mathrm{P}_{\mathrm{CO}_{2}}$ levels (one-way ANOVA, $F=484$, df $=2, p<0.001$; Tukey Post hoc, $\mathrm{df}=2$, $p<0.001$ ) (Table 4).

\section{PIC : POC ratio and POC : PON ratio}

Both, changing carbonate chemistry conditions and light intensity independently and interactively affected PIC : POC ratio and POC : PON ratio (Table 2). At each light treatment, PIC : POC ratios at $51 \mathrm{~Pa} \mathrm{P}_{\mathrm{CO}_{2}}$ were significantly higher than at 105 and $152 \mathrm{~Pa} \mathrm{P}_{\mathrm{CO}_{2}}$ (Tukey Post hoc, $\mathrm{df}=1, p<0.05$ ) (Fig. 3E). Significant differences in PIC : POC ratios between $105 \mathrm{~Pa}$ and $152 \mathrm{~Pa} \mathrm{P}_{\mathrm{CO}_{2}}$ were found at $100 \mu \mathrm{mol}$ photons $\mathrm{m}^{-2} \mathrm{~s}^{-1}, 400 \mu \mathrm{mol}$ photons $\mathrm{m}^{-2} \mathrm{~s}^{-1}$, and $600 \mu \mathrm{mol}$ photons $\mathrm{m}^{-2} \mathrm{~s}^{-1}$ (Tukey Post hoc, all $\mathrm{df}=1$, all $p<0.01$ ). There was no obvious trend between PIC : POC ratio and light intensity.

Both, elevated $\mathrm{P}_{\mathrm{CO}_{2}}$ and higher light intensity reduced POC : PON ratios (Tukey Post hoc, $\mathrm{df}=1, p>0.05$ ) (Fig. 3F). At $51 \mathrm{~Pa} \mathrm{P}_{\mathrm{CO}_{2}}$, POC : PON ratios at $50 \mu \mathrm{mol}$ photons $\mathrm{m}^{-2}$ $\mathrm{s}^{-1}$ and $100 \mu \mathrm{mol}$ photons $\mathrm{m}^{-2} \mathrm{~s}^{-1}$ were slightly higher than at other treatment conditions (Tukey Post hoc, all $p>0.05$ ) (Table 3).

\section{Discussion}

Changing carbonate chemistry modulates the light responses of photosynthetic carbon fixation, calcification and growth rates

POC production and growth rates of marine phytoplankton usually increase with increasing light intensity, level off at saturating light intensities and then decline again at inhibiting light levels (e.g., Geider et al. 1997; Gao et al. 2012; Fig. 4A). By fitting the light response curve given in Eq. 2 to our data, we found that rising $\mathrm{P}_{\mathrm{CO}_{2}}$ reduced the maximum rates of growth and photosynthetic carbon fixation (Figs. 1A, 3A; Table 4). Presumably, rising $\mathrm{P}_{\mathrm{CO}_{2}}$ could reduce the need for CCM activity thereby saving energy (Raven 1991; Hopkinson et al. 2011; McCarthy et al. 2012). In our case, the lower potential for energy dissipation toward higher $\mathrm{P}_{\mathrm{CO}_{2}}$ (lower CCM activity) may exacerbate photo inhibition thereby explaining the reduced growth and POC production rates (Figs. 1, 3).

Another reason for higher growth and POC production rates at lower $\mathrm{P}_{\mathrm{CO}_{2}}$ and increasing light intensities (Figs. 1B, 3B) may be that increasing light intensities facilitate the operation of CCMs (Rokitta and Rost 2012) thereby enabling cells to satisfy their inorganic carbon requirements for POC 

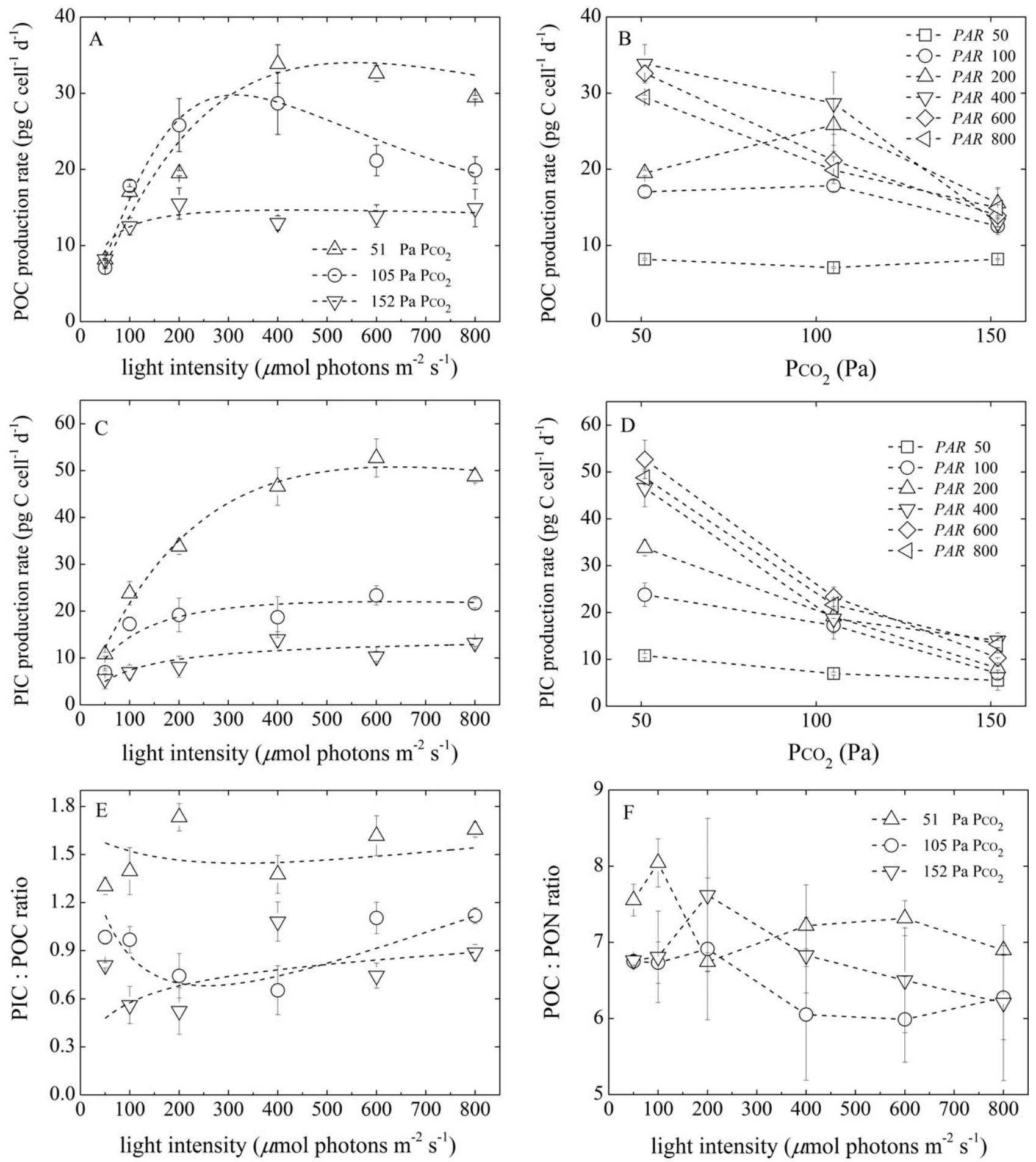

Fig. 3. Effects of light intensity and $\mathrm{P}_{\mathrm{CO}_{2}}$ level on $\mathrm{POC}$ and $\mathrm{PIC}$ production rates, $\mathrm{PIC}$ : $P O C$ ratio and POC : PON ratio of $G$. oceanica. Panels (A), (C), (E), (F) depict POC production rate, PIC production rate, PIC : POC ratio and POC : PON ratio as a function of light intensities at $51(\triangle), 105(\bigcirc)$ and $152(\nabla) \mathrm{Pa} \mathrm{P}_{\mathrm{CO}_{2}}$. Panels (B), (D) show POC production rate and PIC production rate as a function of $\mathrm{P}_{\mathrm{CO}_{2}}$ at $50 \mu \mathrm{mol}$ photons $\mathrm{m}^{-2} \mathrm{~s}^{-1}, 100$

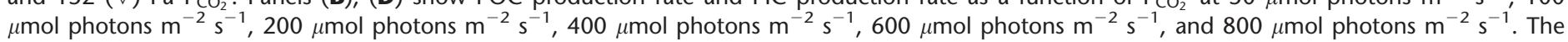
values represent the mean of four replicates, with error bars representing \pm one standard deviation. 


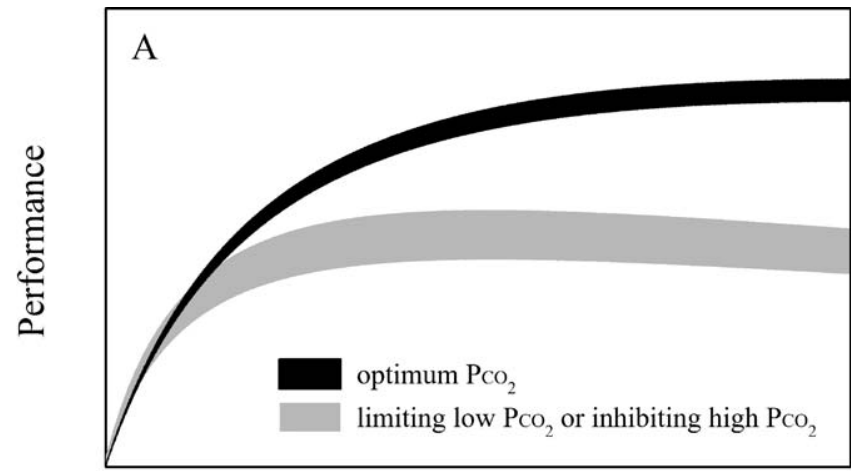

light intensity

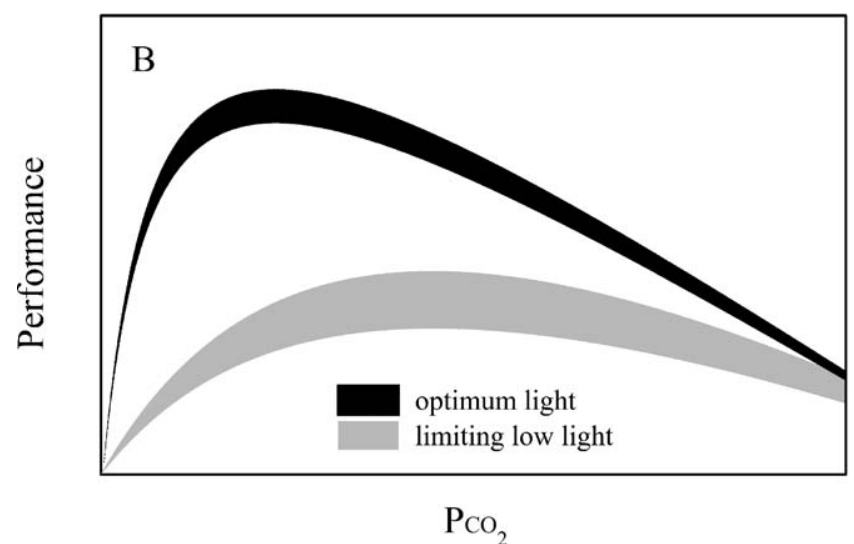

Fig. 4. Conceptual drawing for the interactive effects of light intensity and $\mathrm{P}_{\mathrm{CO}_{2}}$ level on the performance (representing growth, photosynthetic carbon fixation, and calcification) of G. oceanica. (A) The modulating effect of $\mathrm{P}_{\mathrm{CO}_{2}}$ on the light response curve. Maximum rates at limiting low $\mathrm{P}_{\mathrm{CO}_{2}}$ and inhibiting high $\mathrm{P}_{\mathrm{CO}_{2}}$ are lower than at optimum $\mathrm{P}_{\mathrm{CO}_{2}}$. (B) The modulating effect of light intensity on the $\mathrm{P}_{\mathrm{CO}_{2}}$ sensitivity. Limiting low light intensity shifts the $\mathrm{P}_{\mathrm{CO}_{2}}$ optimum toward higher level and reduces the maximum rate.

production and growth rates already at lower $\mathrm{P}_{\mathrm{CO}_{2}}$ levels. In fact, at high light intensity, high $\mathrm{P}_{\mathrm{CO}_{2}}$ grown cells were found to possess less photosystem I (PS1) per cell and keep a smaller proportion of PSII complexes open compared with low $\mathrm{P}_{\mathrm{CO}_{2}}$ grown cells (Burns et al. 2006). Lower PSI and PSII absorbance capacities for light in high $\mathrm{P}_{\mathrm{CO}_{2}}$ grown cells could also be expected to lead to lower POC production and growth rates. Furthermore, the proton concentration $\left(\left[\mathrm{H}^{+}\right]\right)$ in the cytosol of the coccolithophore Emiliania huxleyi was found to increase with increasing $\left[\mathrm{H}^{+}\right]$in seawater (Suffrian et al. 2011). Cells growing at high $\mathrm{P}_{\mathrm{CO}_{2}}$ could suffer from the negative effect of high $\left[\mathrm{H}^{+}\right]$on POC production (Bach et al. 2011) even more so as this effect may be exacerbated by high light intensities (Ihnken et al. 2011).

Calcification is an energy-dependent process and often reduced in coccolithophores at $\mathrm{P}_{\mathrm{CO}_{2}}$ levels projected for the end of this century (Riebesell and Tortell 2011; Meyer and Riebesell 2015). However, only some studies focussed on the interactive effects of $\mathrm{CO}_{2}$ concentration and light intensity on coccolithophore calcification (Zondervan et al. 2002; Feng et al. 2008; Gao et al. 2009; Rokitta and Rost 2012). In this study, a increasing light intensity accelerated calcification rates strongly at lower $\mathrm{P}_{\mathrm{CO}_{2}}$ whereas this positive effect was absent at higher $\mathrm{P}_{\mathrm{CO}_{2}}$ (Fig. 3C), similar to growth and photosynthetic carbon fixation rates. This supports earlier findings that the sensitivity of calcification rates to light intensity can be modulated by carbonate chemistry (Zondervan et al. 2002; Feng et al. 2008; Gao et al. 2009; Rokitta and Rost 2012). The underlying physiological explanation could be that at limiting light intensity, light is the dominant factor determining the calcification rate and differences in carbonate chemistry conditions are presumably less important. This can be seen at the lowest light intensity in this study (50 $\mu \mathrm{mol}$ photons $\left.\mathrm{m}^{-2} \mathrm{~s}^{-1}\right)$, where PIC production rates did not show a significant difference among the three $\mathrm{P}_{\mathrm{CO}_{2}}$ treatments (Fig. 3D). At saturating light intensity, however, differences in $\mathrm{CO}_{2}$ or $\mathrm{H}^{+}$apparently induce a significant effect on calcification rates (Feng et al. 2008).

For $\mathrm{P}_{\mathrm{CO}_{2}}$ levels lower than $29 \mathrm{~Pa}$, Sett et al. (2014) concluded that POC and PIC production and growth rates in the same G. oceanica strain were limited by inorganic carbon availability. Although such low $\mathrm{P}_{\mathrm{CO}_{2}}$ levels were not included in our study, by fitting the light response curves (Figs. 1A, $3 \mathrm{~A}, \mathrm{C}$ ) we conclude that both limiting low $\mathrm{P}_{\mathrm{CO}_{2}}$ and inhibiting high $\mathrm{P}_{\mathrm{CO}_{2}}$ levels reduce the maximum values for photosynthetic carbon fixation, calcification and growth rates of coccolithophores (Fig. 4A; Table 4).

Rising $\mathrm{P}_{\mathrm{CO}_{2}}$ and increasing light intensity synergistically alter the electron transport rate in the light reaction

rETR is a measure for photosynthetic efficiency (Schreiber et al. 1995). To acclimate to high irradiances, phytoplankton cells regulate the photosystem stoichiometry (PSI : PSII) by lowering the amount of photosystem I (PSI) reaction centers relative to PSII complexes (Sonoike et al. 2001). Here we found that the $\mathrm{rETR}_{\max }$ response of G. oceanica (Fig. 2A) to high light intensities was depending on the incubation $\mathrm{P}_{\mathrm{CO}_{2}}$ which implies that different $\mathrm{P}_{\mathrm{CO}_{2}}$ levels induced changes in PSI : PSII. A study by Burns et al. (2006) revealed that at high light intensity, low $\mathrm{P}_{\mathrm{CO}_{2}}$ grown cells contained significantly more PsaC protein (core subunit of photosystem I) in the PSI complex than high $\mathrm{P}_{\mathrm{CO}_{2}}$ grown cells. Furthermore, Burns et al. (2006) found that across the range of growth irradiances, PsaC : PSII absorbance capacity (an indicator of PSI content relative to the capacity of PSII to capture light energy) increased in low $\mathrm{P}_{\mathrm{CO}_{2}}$ grown cells, whereas they slightly decreased in high $\mathrm{P}_{\mathrm{CO}_{2}}$ grown cells. Thus, the observed reduction in $\mathrm{rETR}_{\max }$ with increasing $\mathrm{P}_{\mathrm{CO}_{2}}$ at high light intensities may be due to lower PSI : PSII.

Phytoplankton can alter light absorption for photosynthesis to enable acclimation over a wide range of irradiances (Henriksen et al. 2002). Algae tend to reduce the 
pigment contents such as chlorophyll $a$, carotenoids or fucoxanthin in antenna systems to prevent excessive energy to be transferred to PSII reaction centres (Henriksen et al. 2002; Barcelos e Ramos et al. 2012). At $50 \mu \mathrm{mol}$ photons $\mathrm{m}^{-2} \mathrm{~s}^{-1}$ in this study, the quantum yield of PSII $\left(F_{\mathrm{v}} / F_{\mathrm{m}}\right.$, the ratio of photons transferred in the ETR to photons absorbed by PSII) at $152 \mathrm{~Pa}$ was only 6\% lower than at $51 \mathrm{~Pa}$. However, at $800 \mu \mathrm{mol}$ photons $\mathrm{m}^{-2} \mathrm{~s}^{-1}$ the quantum yield of PSII at $152 \mathrm{~Pa}$ was 30\% lower than at $51 \mathrm{~Pa}$ (data not shown). This indicates that high light intensity and high $\mathrm{P}_{\mathrm{CO}_{2}}$ may synergistically reduce the quantum yield of PSII (the maximum efficiency of PSII), which leads to large diminution in alpha at high $\mathrm{P}_{\mathrm{CO}_{2}}$ and high light conditions (Fig. 2B).

Light intensity modulates the $\mathrm{P}_{\mathrm{CO}_{2}}$ sensitivity of photosynthetic carbon fixation, calcification and growth rates

Physiological responses to a broad range of $\mathrm{P}_{\mathrm{CO}_{2}}$ levels of coccolithophores investigated in this respect so far displayed optimum curve patterns (Langer et al. 2006; Bach et al. 2011, 2015; Sett et al. 2014; Müller et al. 2015). Growth and production rates of POC and PIC increase with increasing $\mathrm{P}_{\mathrm{CO}_{2}}$ levels, reach a maximum, and then decline linearly with further $\mathrm{P}_{\mathrm{CO}_{2}}$ (proton concentration $\left(\left[\mathrm{H}^{+}\right]\right)$) increase (Bach et al. 2011; Sett et al. 2014; Bach et al. 2015; Fig. 4B). The $\mathrm{CO}_{2}$ and $\mathrm{HCO}_{3}^{-}$availability was identified as the factor responsible for the observed decline of growth and production rates toward the left side of the optimum, the proton concentration $\left(\left[\mathrm{H}^{+}\right]\right)$was the driving factor toward the right side of the optimum (Bach et al. 2011, 2015). The sensitivities of these rates to inorganic carbon availability and $\mathrm{H}^{+}$ were clearly modulated by light intensity (Figs. 1B, 3B,D). Light availability is likely to affect the supply of inorganic carbon to photosynthesis, calcification and growth in general (Zondervan et al. 2002; Barcelos e Ramos et al. 2012; Rokitta and Rost 2012).

We did not directly determine the $\mathrm{P}_{\mathrm{CO}_{2}}$ optimum for PIC production rates in this study as our treatment levels were limited. Sett et al. (2014), however, found that the $\mathrm{P}_{\mathrm{CO}_{2}}$ optimum for calcification in the same $G$. oceanica strain was at about $29 \mathrm{~Pa}$ at $20^{\circ} \mathrm{C}$ and $150 \mu \mathrm{mol}$ photons $\mathrm{m}^{-2} \mathrm{~s}^{-1}$. According to this, the lowest $\mathrm{P}_{\mathrm{CO}_{2}}$ level applied in our study $(51 \mathrm{~Pa})$ was too high to detect the optimum of the G. oceanica $\mathrm{P}_{\mathrm{CO}_{2}}$ response curve. Nevertheless, assuming an optimum curve response and using the model described in Bach et al. (2011) as given in Eq. 8, the optimum $\mathrm{P}_{\mathrm{CO}_{2}}$ for photosynthetic carbon fixation, calcification and growth rates shift toward lower levels with increasing light intensities (Figs. 1B, 3B). This is in line with findings by Rokitta and Rost (2012) who showed that the half-saturation DIC concentrations for carbon fixation of the calcifying algae E. huxleyi were $111 \mu \mathrm{mol}$ $\mathrm{kg}^{-1}$ at $50 \mu \mathrm{mol}$ photons $\mathrm{m}^{-2} \mathrm{~s}^{-1}$ and $20 \mu \mathrm{mol} \mathrm{kg}{ }^{-1}$ at 300 $\mu$ mol photons $\mathrm{m}^{-2} \mathrm{~s}^{-1}$. The reasons for the shift could be

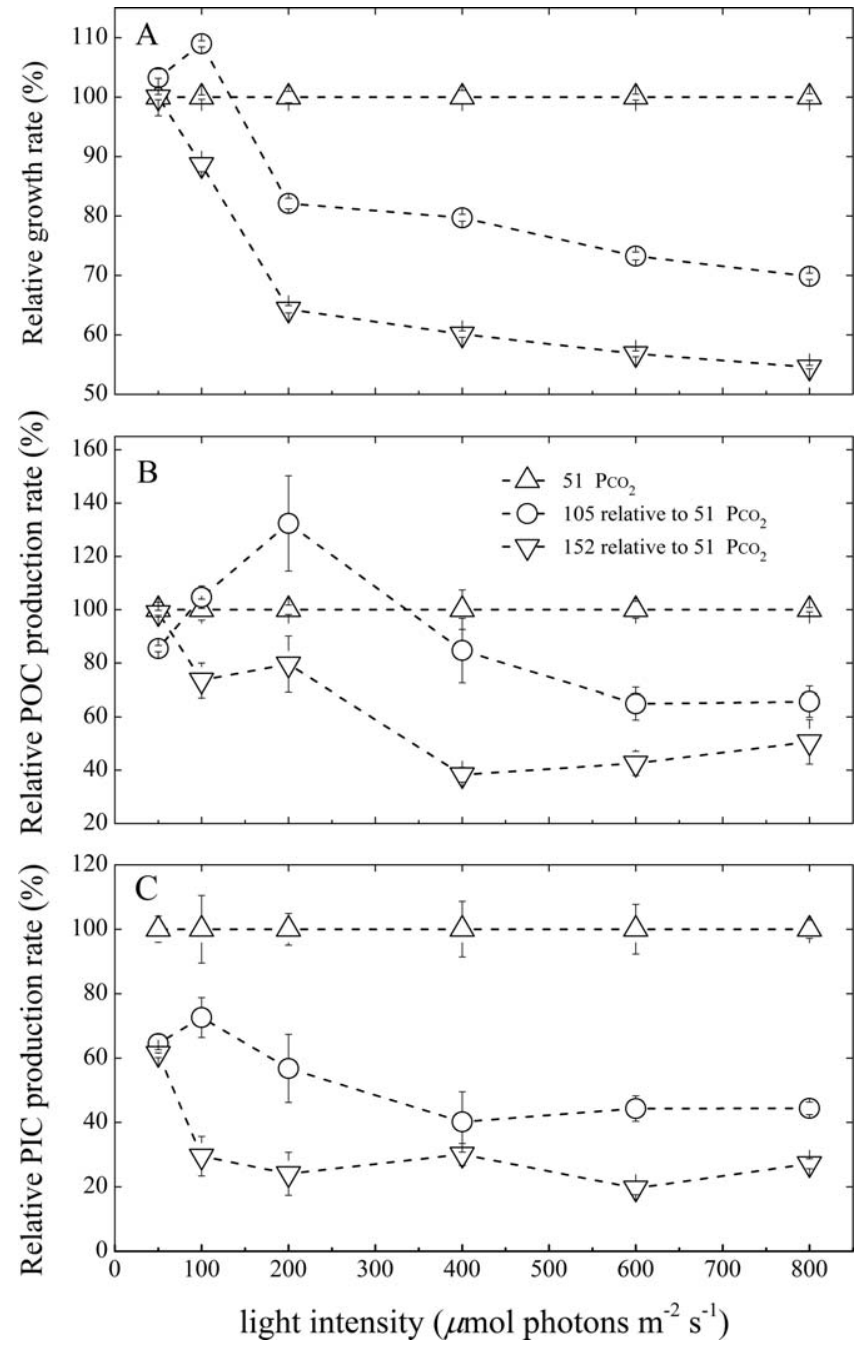

Fig. 5. Comparison of growth, $\mathrm{POC}$ and $\mathrm{PIC}$ production rates at 105 $(\bigcirc)$ and $152(\nabla) \mathrm{Pa}_{\mathrm{CO}_{2}}$ relative to those at $51(\triangle) \mathrm{Pa} \mathrm{P}_{\mathrm{CO}_{2}}$. The values represent the mean of four replicates, with error bars representing \pm one standard deviation.

that higher light intensities provide more energy to allow for higher CCM activity which would help to overcome carbon limitation at lower $\mathrm{P}_{\mathrm{CO}_{2}}$ levels (McGinn et al. 2003; Rokitta and Rost 2012). Following the growth rate response to light intensity shown in Fig. 1A, growth rate is expected to decrease toward increasingly inhibiting high light intensities beyond the measurement range of $800 \mu \mathrm{mol}$ photons $\mathrm{m}^{-2}$ $\mathrm{s}^{-1}$. If growth rates would drop more pronounced at low than at high $\mathrm{P}_{\mathrm{CO}_{2}}$ levels, the $\mathrm{P}_{\mathrm{CO}_{2}}$ optimum for growth shown in Fig. 1B is expected to be higher at inhibiting high light than at optimum light intensity.

Based on the results of this and other studies (Zondervan et al. 2002; Rost et al. 2003), we conclude that the optimum $\mathrm{P}_{\mathrm{CO}_{2}}$ levels for growth, POC and PIC production rates at limiting low light are higher than at optimum light intensities (Figs. 1B, 3B,D, 4B). Furthermore, the maximum values for 
these rates are lower at limiting low light than at optimum light intensities (Fig. 4B).

In an earlier study with E. huxleyi, Rokitta and Rost (2012) put forward a light dependent model suggesting that the rate of a physiological process is governed primarily by light intensity, and changes in $\mathrm{P}_{\mathrm{CO}_{2}}$ levels will exacerbate or weaken the effects of light intensity on this rate. Our data supported the light dependent model (Figs. 1, 3). However, Rokitta and Rost (2012) found that the sensitivities of POC and PIC production rates of E. huxleyi to high $\mathrm{P}_{\mathrm{CO}_{2}}$ are stronger at $50 \mu \mathrm{mol}$ photons $\mathrm{m}^{-2} \mathrm{~s}^{-1}$ than at $300 \mu$ mol photons $\mathrm{m}^{-2} \mathrm{~s}^{-1}$, whereas we observed a stronger effect of high $\mathrm{P}_{\mathrm{CO}_{2}}$ on POC and PIC production rates at $600-800 \mu \mathrm{mol}$ photons $\mathrm{m}^{-2} \mathrm{~s}^{-1}$ compared with $50 \mu \mathrm{mol}$ photons $\mathrm{m}^{-2} \mathrm{~s}^{-1}$ (Fig. 5). This discrepancy is possibly due to the variable sensitivities of E. huxleyi and G. oceanica to light and $\mathrm{P}_{\mathrm{CO}_{2}}$.

The results of our study show that increasing light intensity decreases the $\mathrm{P}_{\mathrm{CO}_{2}}$ optima for carbon fixation, calcification and growth rates of $G$. oceanica. In contrast, rising temperature had the opposite effect, increasing the $\mathrm{P}_{\mathrm{CO}_{2}}$ optima for these rates (Sett et al. 2014). These opposing trends are likely due to the fact that temperature primarily modulates carbon demand by accelerating metabolic activity, whereas light also affects carbon supply through energy provision to carbon uptake mechanisms. In the future ocean, both light intensity and temperature in the upper mixed layer will generally increase (Sarmiento et al. 2004; IPCC 2013). How these combined effects will affect the competitive fitness of this and other coccolithophore species under future ocean scenarios is difficult to predict with the information presently available (Riebesell and Gattuso 2015). It emphasizes the need for further investigation on the interacting effect of simultaneous modification of life-sustaining properties such as temperature, $\mathrm{CO}_{2}$, light, and nutrients in the marine environment.

\section{References}

Bach, L. T., U. Riebesell, and K. G. Schulz. 2011. Distinguishing between the effects of ocean acidification and ocean carbonation in the coccolithophore Emiliania huxleyi. Limnol. Oceanogr. 56: 2040-2050. doi:10.4319/lo.2011.56.6.2040

Bach, L. T., U. Riebesell, M. A. Gutowska, L. Federwisch, and K. G. Schulz. 2015. A unifying concept of coccolithophore sensitivity to changing carbonate chemistry embedded in an ecological framework. Prog. Oceanogr. 135: 125-138. doi:10.1016/j.pocean.2015.04.012

Barcelos e Ramos, J., K. G. Schulz, S. Febiri, and U. Riebesell. 2012. Photoacclimation to abrupt changes in light intensity by Phaeodactylum tricornutum and Emiliania huxleyi: The role of calcification. Mar. Ecol. Prog. Ser. 452: 11-26. doi:10.3354/meps09606

Björkman, O., and B. Demmig. 1987. Photon yield of $\mathrm{O}_{2}$ evolution and chlorophyll fluorescence characteristics at
$77 \mathrm{~K}$ among vascular plants of diverse origins. Planta 170: 489-504. doi:10.1007/BF00402983

Bopp, L., and others. 2001. Potential impact of climate change on marine export production. Global Biogeochem. Cycles 15: 81-99. doi:10.1029/1999GB001256

Braarud, T., K. R. Gaarder, J. Marklaki, and E. Nordli. 1952. Coccolithophorids studied in the electron microscope. Observations on Coccolithus huxleyi and Syracosphaera carterae. Nytt Mag. Bot. 1: 129-134.

Burns, R. A., T. D. B. MacKenzie, and D. A. Campbell. 2006. Inorganic carbon repletion constrains steady-states light acclimation in the cyanobacterium Synechococcus elongatus. J. Phycol. 42: 610-621. doi:10.1111/j.1529-8817.2006.00220.x

Danbara, A., and Y. Shiraiwa. 1999. The requirement for selenium for the growth of marine coccolithophorids, Emiliania huxleyi, Gephyrocapsa oceanica and Helladosphaera sp. (Prymnewsiophyceae). Plant Cell Physiol. 40: 762-766. doi:10.1093/oxfordjournals.pcp.a029603

Dickson, A. G., J. D. Afghan, and G. C. Anderson. 2003. Reference materials for oceanic $\mathrm{CO}_{2}$ analysis: A method for the certification of total alkalinity. Mar. Chem. 80: 185197. doi:10.1016/S0304-4203(02)00133-0

Eilers, P., and J. Peeters. 1988. A model for the relationship between light intensity and the rate of photosynthesis in phytoplankton. Ecol. Model. 42: 199-215. doi:10.1016/ 0304-3800(88)90057-9

Feng, Y., M. E. Warner, Y. Zhang, J. Sun, F. X. Fu, J. M. Rose, and D. A. Hutchins. 2008. Interactive effects of increased $\mathrm{P}_{\mathrm{CO}_{2}}$, temperature and irradiance on the marine coccolithophore Emiliania huxleyi (Prymnesiophyceae). Eur. J. Phycol. 43: 87-98. doi:10.1080/09670260701664674

Gao, K., Z. Ruan, V. E. Villafañe, J. P. Gattuso, and E. W. Helbling. 2009. Ocean acidification exacerbates the effect of UV radiation on the calcifying phytoplankton Emiliania huxleyi. Limnol. Oceanogr. 54: 1855-1862. doi:10.4319/ lo.2009.54.6.1855

Gao, K., and others. 2012. Rising $\mathrm{CO}_{2}$ and increased light exposure synergistically reduce marine primary productivity. Nat. Clim. Change 2: 519-523. doi:10.1038/nclimate1507

Geider, R. J., H. L. MacIntyre, and T. M. Kana. 1997. Dynamic model of phytoplankton growth and acclimation: Responses of the balanced growth rate and the chlorophyll a: carbon ratio to light, nutrient-limitation and temperature. Mar. Ecol. Prog. Ser. 148: 187-200. doi:10.3354/meps148187

Giordano, M., J. Beardall, and J. A. Raven. 2005. CO 2 concentrating mechanisms in algae: Mechanisms, environmental modulation and evolution. Annu. Rev. Plant Biol. 56: 99131. doi:10.1146/annurev.arplant.56.032604.144052

Guillard, R. R., and J. H. Ryther. 1962. Studies of marine planktonic diatoms. I. Cyclotella nana Hustedt, and Detonula confervacea (cleve) Gran. Can. J. Microbiol. 8: 229239. doi:10.1139/m62-029

Henriksen, P., B. Riemann, H. Kaas, H. M. Sørensen, and H. L. Sørensen. 2002. Effects of nutrient-limitation and 
irradiance on marine phytoplankton pigments. J. Plankton Res. 24: 835-858. doi:10.1093/plankt/24.9.835

Hopkinson, B. M., C. L. Dupont, A. E. Allen, and F. M. M. Morel. 2011. Efficiency of the $\mathrm{CO}_{2}$-concentrating mechanism of diatoms. Proc. Natl. Acad. Sci. USA 8: 3830-3837. doi:10.1073/pnas.1018062108

Houghton, J. T., and others. 2001. Climate change 2001: The scientific basis, p. 36-40. Cambridge Univ. Press.

Ihnken, S., S. Roberts, and J. Beardall. 2011. Differential responses of growth and photosynthesis in the marine diatom Chaetoceros muelleri to $\mathrm{CO}_{2}$ and light availability. Phycologia 50: 182-193. doi:10.2216/10-11.1

Intergovernmental Panel on Climate Change (IPCC). 2013. Summary for Policymakers, p: 3-33. In T. F. Stocker, and others [eds.], Climate change 2013: The physical science basis. Contribution of Working Group I to the Fifth Assessment Report of the Intergovernmental Panel on Climate Change. Cambridge Univ. Press.

Kaeriyama, H., F. Katsuki, M. Otsubo, M. Yamada, K. Ichimi, K. Tada, and P. J. Harrison. 2011. Effects of temperature and irradiance on growth of strains belonging to seven Skeletonema species isolated from Dokai Bay, southern Japan. Eur. J. Phycol. 46: 113-124. doi:10.1080/09670262.2011.565128

Kester, D., I. W. Duedall, D. N. Connors, and R. M. Pytkowicz. 1967. Preparation of artificial seawater. Limnol. Oceanogr. 1: 176-179. doi:10.4319/lo.1967.12.1.0176

Langer, G., M. Geisen, K. H. Baumann, J. Kläs, U. Riebesell, S. Thoms, and J. R. Young. 2006. Species-specific responses of calcifying algae to changing seawater carbonate chemistry. Geochem. Geophys. Geosyst. 7: 1-12. doi: 10.1029/2005GC001227

McCarthy, A., S. P. Rogers, S. J. Duffy, and D. A. Campbell. 2012. Elevated carbon dioxide differentially alters the photophysiology of Thalassiosira pseudonana (Bacillariophyceae) and Emiliania huxleyi (Haptophyta). J. Phycol. 48: 635-646. doi:10.1111/j.1529-8817.2012.01171.x

McGinn, P. J., G. D. Price, R. Maleszka, and M. R. Badger. 2003. Inorganic carbon limitation and light control the expression of transcripts related to the $\mathrm{CO}_{2}$-concentrating mechanism in the cyanobacterium Synechocystis sp. strain PCC 6803. Plant Physiol. 132: 218-229. doi:10.1104/pp.102.019349

Merico, A., T. Tyrrell, E. J. Lessard, T. Oguz, P. J. Stabeno, S. I. Zeeman, and T. E. Whitledge. 2004. Modelling phytoplankton succession on the Bering Sea shelf: Role of climate influences and trophic interactions in generating Emiliania huxleyi blooms 1997-2000. Deep-Sea Res. Part I 51: 1803-1826. doi:10.1016/j.dsr.2004.07.003

Meyer, J., and U. Riebesell. 2015. Reviews and syntheses: Responses of coccolithophores to ocean acidification: A meta-analysis. Biogeosciences 12: 1671-1682. doi: 10.5194/bg-12-1671-2015

Müller, M. N., T. W. Trull, and G. M. Hallegraeff. 2015. Different responses of three Southern Ocean Emiliania huxleyi ecotypes to changing seawater carbonate chemistry. Mar. Ecol. Prog. Ser. 531: 81-90. doi:10.3354/meps11309
Pierrot, D., E. Lewis, and D. W. R. Wallace. 2006. MS Excel program developed for $\mathrm{CO}_{2}$ system calculations. ORNL/ CDIAC-105. Carbon Dioxide Information Analysis Centre, Oak Ridge National Laboratory, U.S. Department of Energy. doi:10.3334/CDIAC/otg.CO2SYS_XLS_CDIAC105a

Raven, J. A. 1991. Physiology of inorganic C acquisition and implications for resource use efficiency by marine phytoplankton: Relation to increased $\mathrm{CO}_{2}$ and temperature. Plant Cell Environ. 14: 779-794. doi:10.1111/j.13653040.1991.tb01442.x

Riebesell, U. 2004. Effects of $\mathrm{CO}_{2}$ enrichment on marine phytoplankton. J. Oceanogr. 60: 719-729. doi:10.1007/ s10872-004-5764-Z

Riebesell, U., and P. D. Tortell. 2011. Effects of ocean acidification on pelagic organisms and ecosystems, p. 99-121. In J. P. Gattuso and L. Hansson [eds.], Ocean acidification. Oxford Univ. Press.

Riebesell, U., and J. P. Gattuso. 2015. Lessons learned from ocean acidification research. Nat. Clim. Chang. 5: 12-14. doi:10.1038/nclimate2456

Rokitta, S. D., and B. Rost. 2012. Effects of $\mathrm{CO}_{2}$ and their modulation by light in the life-cycle stages of the coccolithophore Emiliania huxleyi. Limnol. Oceanogr. 57: 607618. doi:10.4319/lo.2012.57.2.0607

Rost, B., U. Riebesell, and S. Burkhardt. 2003. Carbon acquisition of bloom-forming marine phytoplankton. Limnol. Oceanogr. 48: 56-67. doi:10.4319/lo.2003.48.1.0055

Rost, B., and U. Riebesell. 2004. Coccolithophores and the biological pump: Responses to environmental changes, p. 99125. In H. R. Thierstein and J. R. Young [eds.], Coccolithophores: From molecular biology to global impact. Springer.

Roy, R. N., and others. 1993. Thermodynamics of the dissociation of boric acid in seawater at S 535 from 0 degrees C to 55 degrees C. Mar. Chem. 44: 243-248. doi:10.1016/ 0304-4203(93)90206-4

Sabine, C. L., and others. 2004. The oceanic sink for anthropogenic $\mathrm{CO}_{2}$. Science 305: 367-371. doi:10.1126/ science. 1097403

Sarmiento, J. L., and others. 2004. Response of ocean ecosystems to climate warming. Global Biogeochem. Cycle 18: GB3003. doi:10.1029/2003GB002134

Schippers, P., M. Lürling, and M. Scheffer. 2004. Increase of atmospheric $\mathrm{CO}_{2}$ promotes phytoplankton productivity. Ecol. Lett. 7: 446-451. doi:10.1111/j.1461-0248.2004.00597.x

Schreiber, U., W. Bilger, and C. Neubauer. 1995. Chlorophyll fluorescence as a nonintrusive indicator for rapid assessment of in vivo photosynthesis, p. 49-70. In E. D. Schulze and M. M. Caldwell [eds.], Ecophysiology of photosynthesis. Springer.

Sett, S., L. T. Bach, K. G. Schulz, S. Koch-Klavsen, M. Lebrato, and U. Riebesell. 2014. Temperature modulates coccolithophorid sensitivity of growth, photosynthesis and calcification to increasing seawater $\mathrm{P}_{\mathrm{CO}_{2}}$. PLoS ONE 9: e88308. doi:10.1371/journal.pone.0088308 
Sonoike, K., Y. Hihara, and M. Ikeuchi. 2001. Physiological significance of the regulation of photosystem stoichiometry upon high light acclimation of Synechocystis sp. PCC 6803. Plant Cell Physiol. 42: 379-384. doi:10.1093/pcp/ pce046

Suffrian, K., K. G. Schulz, M. A. Gutowska, U. Riebesell, and M. Bleich. 2011. Cellular pH measurements in Emiliania huxleyi reveal pronounced membrane proton permeability. New Phytol. 190: 595-608. doi:10.1111/j.14698137.2010.03633.x

Xu, K., and K. S. Gao. 2012. Reduced calcification decreases photoprotective capability in the coccolithophorid Emiliania huxleyi. Plant Cell Physiol. 53: 1267-1274. doi: 10.1093/pcp/pcs066

Zondervan, I., B. Rost, and U. Riebesell. 2002. Effect of $\mathrm{CO}_{2}$ concentration on the PIC/POC ratio in the coccolithophore Emiliania huxleyi grown under light-limiting condi- tions and different day lengths. J. Exp. Mar. Biol. Ecol. 272: 55-70. doi:10.1016/S0022-0981(02)00037-0

\section{Acknowledgments}

The authors are grateful to Silke Lischka for reading and revising the manuscript, to Andrea Ludwig and Jana Meyer for dissolved inorganic carbon measurements, and to Kerstin Nachtigall for particulate organic and inorganic carbon measurements. We also thank the China Scholarship Council (CSC) for its support of Yong Zhang. This work was supported by the German Federal Ministry of Education and Research (Bundesministerium für Bildung und Forschung) in the framework of the collaborative project Biological Impacts of Ocean Acidification (BIOACID). Kai G. Schulz is the recipient of an Australian Research Council Future Fellowship (FT120100384).

Submitted 25 February 2015 Revised 29 July 2015

Accepted 3 August 2015

Associate editor: Heidi Sosik 